\title{
ETHICS OF GENETIC COUNSELING IN EPILEPSY
}

The ethical issues raised by families involved with epilepsy are reviewed from the Faculty of Medicine and Research Centre for Public Law, Universite de Montreal, Canada; and the Foundation for Genetic Medicine, Reston, MD. The mode of inheritance in most cases of epilepsy is complex, with genetic heterogeneity and variable expression. The clinical uses of genetic testing include medical management, diagnosis, prevention, and determination of risk to future children in family planning. Genetic research facilitates the recognition of new epilepsy syndromes and the development of new antiepileptic drugs. From an ethical viewpoint, the potential benefits of diagnosis and treatment must be weighed against the potential harm of genetic labeling. Genetic testing should be performed only if it promotes the wellbeing of the individual. The risks of guilt, blame, stigmatization, and discrimination emphasize the need for further research on the ethical issues raised by genetic testing. Genetic counselors must advise patients and families of the economic and social risks of participating in genetic research and genetic testing. The multifactorial nature of epilepsy etiology and our limited understanding of the disorder can foster misinterpretation of the genetic findings by insurers and employers and lead to unwanted financial and emotional hardships. (Godard B, Cardianal G. Ethical implications in genetic counseling and family studies of the epilepsies. Epilepsy \& Behav 2004;5:621-626). (Respond: E-mail: Beatrice.godard@umontreal.ca).

COMENT. The recommended approach to genetic counseling concerning epilepsy is a case-by-case determination of the benefit and harm of testing and the need to protect the individual privacy and wellbeing.

ANKH gene, autosomal dominant early childhood seizures and chondrocalcinosis. ANKH is added to the list of genes involved in idiopathic seizure disorders, in a report from Belfast City Hospital; Robert Jones and Agnes Hunt Orthopaedic Hospital, Oswestry, Shropshire; and City Hospital, Nottingham, UK. All members of an English family affected with chondrocalcinosis (CCAL) experienced seizures in early childhood, usually febrile seizures, and they developed recurrent attacks of arthritis and synovitis as adults. A mutation within the ANKH gene on chromosome 5p is associated with CCAL and can predispose to genetic epilepsy and febrile seizures. The seizures are usually benign and resolve by 2 to 4 years of age without neurologic sequelae. The seizure syndrome with CCAL and ANKH gene mutation resembles generalized epilepsy with febrile seizures plus (GEFS+), except that none had seizures after 4 years. ANKH codes for a transmembrane protein involved in regulation of extracellular pyrophosphate ion levels. (McKee $\mathrm{S}$ et al. Epilepsia 2004;45:1258-1260).

\section{EPILEPSY SURGERY FOR TUBEROUS SCLEROSIS}

The surgical amenability of epileptogenic lesions in tuberous sclerosis complex is reviewed in the light of novel presurgical assessment in a report by investigators at University of Rome, Italy; Stanford University, Palo Alto, CA; and Central Illinois Neurohealth Sciences, Bloomington, IL, USA. In carefully selected patients, neurosurgical 